\title{
ORDERING OF OPTIMAL PORTFOLIO ALLOCATIONS IN A MODEL WITH A MIXTURE OF FUNDAMENTAL RISKS
}

\author{
KA CHUN CHEUNG, * University of Calgary \\ HAILIANG YANG, ${ }^{* *}$ The University of Hong Kong
}

\begin{abstract}
In this paper we study a single-period optimal portfolio problem in which the aim of the investor is to maximize the expected utility. We assume that the return of every security in the market is a mixture of some common underlying source of risks. A sufficient condition to order the optimal allocations is obtained, and it is shown that several models studied in the literature before are special cases of the proposed model. In the course of the analysis concepts in stochastic orders are employed, and a new characterization of the likelihood ratio order is obtained.
\end{abstract}

Keywords: Asset allocation; stochastic order; dependence structure; comonotonicity; weak convergence; likelihood ratio order

2000 Mathematics Subject Classification: Primary 62P05

Secondary 60E15

\section{Introduction}

In recent years dependence concepts have attracted attention and are widely studied in various fields. One particular area is the study of optimal portfolio in the classical singleperiod expected utility-based setting. To make the model more realistic, researchers try to abandon the traditional assumption that asset returns are independent. For example, Hennessy and Lapan [7] modeled the dependence structure of asset returns using Archimedean copula. Lapan and Hennessy [10] assumed that the asset returns were location and scale shifts of some permutation symmetric random variables. Cheung and Yang [4] assumed that the assets were subject to dependent default risk. They pointed out that ignoring the dependence structure by merely assuming independence may lead to a wrong rank order of the optimal allocations. In [2] an alternative optimization criterion was suggested. Instead of maximizing the expected utility by fixing the returns' dependence structure, it was suggested that we might first identify the worst dependence structure that would give rise to the minimum expected utility for any fixed allocation, then proceed to study the optimal portfolio problem as if this worst dependence structure were the actual one. The rationale is that a risk-averse investor may adopt a prudent and conservative attitude toward the uncertainty in the dependence structure and, hence, may assume that the dependence structure is most unfavorable to him/her. The advantages of this approach is that we can ignore the complicated modeling issue of the dependence structure altogether, and the worst dependence structure is very often much easier to analyze. This

Received 24 August 2007; revision received 24 November 2007.

* Postal address: Department of Mathematics and Statistics, University of Calgary, Calgary, Alberta, T3A 2E2, Canada. Email address: kccheung@math.ucalgary.ca

** Postal address: Department of Statistics and Actuarial Science, The University of Hong Kong, Pokfulam Road, Hong Kong. 
paradigm has also been applied to other contexts, such as the study of optimal policy limits and deductibles (see [3]).

In this paper we propose a more general dependence model by using a different way to get rid of the independence assumption. We assume that there are some fundamental risks in the market having unknown dependence structure, and the return of each security is simply a mixture of the underlying fundamental risks. Since the return of every security is a mixture on the same group of risks, returns are not independent except in some degenerate cases. Here, the aforementioned worst dependence structure paradigm is applied to deal with the unknown dependence structure among the fundamental risks. While this approach is not modeling the dependence structure explicitly, it has the advantages of being analytically tractable, and being flexible enough to incorporate several optimal portfolio models studied in the literature as special cases.

This paper is organized as follows. The formulation of the problem is presented in Section 2 . The problem of identifying the worst dependence structure among the fundamental risks is solved in Section 3, and the problem of existence is studied in Section 4. In Section 5 we solve the proposed problem by giving a sufficient condition for ordering the optimal allocations. Concepts in the theory of stochastic orders are used heavily. In the course of the analysis we also prove a new characterization of the likelihood ratio order, which enables us to generalize the probabilistic version of the well-known Hardy-Littlewood-Pólya rearrangement inequality presented in [9]. The final section demonstrates the flexibility and the usefulness of our model by showing that it can incorporate some other models studied in the literature as special cases, and our analysis can produce exactly the same results.

We close this introduction by introducing the notation and terminology used in this paper, and briefly reviewing the concept of comonotonicity. All random variables are defined on a common probability space $(\Omega, \mathcal{F}, \mathrm{P})$. Whenever an expectation is mentioned, it is assumed to exist. The usual stochastic order, the convex order, and the increasing concave order are denoted by ' $\leq_{\mathrm{st}}$ ', ' $\leq_{\mathrm{cx}}$ ', and ' $\leq_{\mathrm{icv}}$ ', respectively. A subset $A$ of $\mathbb{R}^{n}$ is said to be comonotonic if whenever $\boldsymbol{x}=\left(x_{1}, \ldots, x_{n}\right)$ and $\boldsymbol{y}=\left(y_{1}, \ldots, y_{n}\right)$ are elements of $A$ then either $x_{i} \leq y_{i}$ for all $i$ or $y_{i} \leq x_{i}$ for all $i$. A random vector $\boldsymbol{X}=\left(X_{1}, \ldots, X_{n}\right)$ in $\mathbb{R}^{n}$ is said to be comonotonic if there is a measurable comonotonic subset $A$ of $\mathbb{R}^{n}$ such that $\mathrm{P}(\boldsymbol{X} \in A)=1$. When a collection of infinitely many random variables is given, we say this collection is comonotonic if any finite subcollection is comonotonic. From the definition, it is clear that if $\left(X_{1}, \ldots, X_{n}\right)$ is comonotonic, and if $g_{1}, \ldots, g_{n}$ are $n$ real-valued functions that are all increasing or all decreasing, then $\left(g_{1}\left(X_{1}\right), \ldots, g_{n}\left(X_{n}\right)\right)$ is also comonotonic. It is also known that $\left(X_{1}, \ldots, X_{n}\right)$ is comonotonic if and only if

$$
\left(X_{1}, \ldots, X_{n}\right) \stackrel{\mathrm{D}}{=}\left(F_{X_{1}}^{-1}(U), \ldots, F_{X_{n}}^{-1}(U)\right)
$$

(where $\stackrel{\text { D }}{=}$ denotes equality in distribution), whenever $U$ is uniformly distributed on $(0,1)$. Here, $F_{X}^{-1}$ is the left-continuous inverse of the distribution function of the random variable $X$. From this equation we can easily deduce that

$$
\mathrm{P}\left(X_{1} \leq s_{2}, \ldots, X_{n} \leq s_{n}\right)=\min \left(\mathrm{P}\left(X_{1} \leq s_{1}\right), \ldots, \mathrm{P}\left(X_{1} \leq s_{1}\right)\right)
$$

for any real numbers $s_{1}, \ldots, s_{n}$. Hence, if a given random vector is known to be comonotonic, its joint distribution can be determined solely from the marginal distributions. Lemma 1, below, is one of the most important results concerning comonotonic random vectors. It states that a comonotonic random vector has the maximum convex sum when the marginal distributions are fixed. An application appears in Section 3. 
Lemma 1. If $\left(X_{1}, \ldots, X_{n}\right)$ and $\left(X_{1}^{\mathrm{c}}, \ldots, X_{n}^{\mathrm{c}}\right)$ have the same marginal distributions, and if the latter is comonotonic, then

$$
X_{1}+\cdots+X_{n} \leq_{\mathrm{cx}} X_{1}^{\mathrm{c}}+\cdots+X_{n}^{\mathrm{c}}
$$

We refer the reader to [5] for a survey of the notion of comonotonicity.

\section{The model}

In the market we assume that there is a group of fundamental risks, which is modeled as a collection of random variables $\mathcal{X}=\left\{X_{j}: j \in J\right\}$ that are probably dependent on each other. Here, $J$ is an arbitrary index set. Extra structures will be imposed on it later. Alternatively, the collection $\mathcal{X}$ can be regarded as a stochastic process indexed by $J$ rather than a collection of random variables. The return of any security in the market is assumed to be a mixture of the fundamental risks, that is, associated with every security is a mixing variable $M$ taking values in $J$ such that the return of the security can be expressed as $X_{M}$.

One possible interpretation of this model is as follows. We may think of $J$ as the collection of all possible investment/economic environments that the underlying companies of the stocks would face. Each possible investment environment $j \in J$ is characterized by an investment return distribution $X_{j}$. Different companies in general have different chances of facing different environments and, hence, can be represented by different mixing variables $M$ taking values in $J$. Obviously, the return of the corresponding stock would be $X_{M}$.

Assume that $n$ securities are available to a risk-averse investor who has an endowment of $W$ dollars, and that the preference of this investor admits a von Neumann-Morgenstern representation. This investor may wish to maximize the expected utility of wealth by investing a suitable amount in each asset. Mathematically, the problem to solve is

$$
\max _{\boldsymbol{a} \in \mathcal{S}(W)} \mathrm{E}\left[U\left(\sum_{i=1}^{n} a_{i} X_{M_{i}}\right)\right],
$$

where $\boldsymbol{a}=\left(a_{1}, \ldots, a_{n}\right)$ represents an admissible portfolio in which $a_{i}$ is the amount invested in the $i$ th security. The maximization is performed over $\delta(W)$, which is defined as the collection of all $n$-tuples of nonnegative numbers whose sum is $W$. The function $U$ is the utility function of the investor, which is assumed to be increasing and concave. The $i$ th security is represented by the mixing variable $M_{i}$ and, hence, its return is $X_{M_{i}}$. Security returns modeled in this way will have a complicated dependence structure, as they are inherited from the dependence structure in $\left\{X_{j}: j \in J\right\}$. The main objective of this paper is to obtain conditions that allow us to rank the optimal allocations to the $n$ securities. However, before any analysis is carried out, more specific assumptions are needed so that the above problem is both meaningful and analytically tractable. Here are the standing assumptions of this paper.

(a) Different random variables in the collection $\mathcal{X}=\left\{X_{j}: j \in J\right\}$ have different distributions. The collection $\mathcal{X}$ is equipped with the weak topology. Moreover, we assume that $X$ can be totally ordered by the usual stochastic order.

(b) The total order on $\mathcal{X}$ induces the corresponding total order on the index set $J$, which will be denoted as ' $\preceq$ '. The set $J$ will be equipped with the topology $g$ that makes $J$ and $\mathcal{X}$ homeomorphic under the canonical bijective map $j \leftrightarrow X_{j}$. The $\sigma$-field on $J$ generated by the topology $\mathcal{g}$ is denoted as $\mathcal{B}(J)$. 
(c) The mixing variables $M_{1}, \ldots, M_{n}$ are independent; each of them is $\mathcal{F} / \mathcal{B}(J)$-measurable. They are also independent of $\left\{X_{j}: j \in J\right\}$.

(d) The process $\left\{X_{j}: j \in J\right\}$ is jointly measurable in the sense that it is $\mathcal{F} \otimes \mathscr{B}(J) / \mathscr{B}(\mathbb{R})$ measurable when viewed as a map from $\Omega \times J$ to $\mathbb{R}$.

(e) The dependence structure among $\left\{X_{j}: j \in J\right\}$ is unknown to the investor. The investor will take a conservative attitude toward this uncertainty by always assuming that the actual dependence structure is the most unfavorable one.

Some explanations are in order. Since our primary objective is to rank the optimal allocations (without specifying the distribution of each $X_{j}$ explicitly), we should be able to compare the 'size' of every pair of underlying risks in the first place. In other words, a total order on $X$ is needed. Recall that a total order is a binary relation that is transitive, antisymmetric, and total. The most natural way to achieve this is to utilize the concept of the usual stochastic order. This leads to the second part in assumption (a). Another justification here is that in our investment/economic environment interpretation of $J$ it is very natural and mathematically desirable to have a ranking among the various environments. This brings us to the total order assumption. Moreover, the best way to compare different environments is to compare their corresponding investment return distributions. This leads to the use of the usual stochastic order. While this order looks strong, it is exactly what our later analysis needs. Without this order, we may not be able to derive a general rule to order the optimal allocations.

While the usual stochastic order is just a partial order on the space of all random variables, we consider a subspace of the space of all random variables, and assume that there is a total order in this subspace. Hence, for every pair of risks $X_{j_{1}}$ and $X_{j_{2}}$, either $F_{j_{1}}(u) \leq F_{j_{2}}(u)$ for all $u \in \mathbb{R}$ or the reversed inequality holds. Here and in the sequel, $F_{j}$ denotes the distribution function of $X_{j}$. If $X_{j_{1}} \leq_{\text {st }} X_{j_{2}}$ but $X_{j_{1}} \neq_{\text {st }} X_{j_{2}}$, we write $X_{j_{1}}<_{\text {st }} X_{j_{2}}$. If $j_{1} \preceq j_{2}$ but $j_{1} \neq j_{2}$, we write $j_{1} \prec j_{2}$.

For further analysis, a topology and a $\sigma$-field structure on $\mathcal{X}$ are needed. Being a collection of random variables, an analytically convenient topology on $\mathcal{X}$ would be the weak topology, which is defined as follows. Let $\mathcal{M}_{1}(\mathbb{R})$ denote the set of all probability measures on $(\mathbb{R}, \mathcal{B}(\mathbb{R}))$, the weak topology on $\mathcal{M}_{1}(\mathbb{R})$ is the weakest topology for which the mapping $\mathcal{M}_{1}(\mathbb{R}) \ni \mu \mapsto$ $\int f \mathrm{~d} \mu$ is continuous for every bounded continuous function $f$ on $\mathbb{R}$. Then the weak topology on $\mathcal{X}$ is simply the relative topology by treating $\mathcal{X}$ as a subset of $\mathcal{M}_{1}(\mathbb{R})$. This explains the first part of assumption (a).

Assumption (b) means that $J$ is equipped with the total order and the topology $g$ through the one to one canonical relationship $\left(j \leftrightarrow X_{j}\right)$ between $J$ and $\mathcal{X}$. Therefore, $(J, \preceq)$ is order isomorphic to $\left(\mathcal{X}, \leq_{\mathrm{st}}\right)$ in that $j_{1} \preceq j_{2}$ if and only if $X_{j_{1}} \leq_{\text {st }} X_{j_{2}}$, and a subset $A$ in $J$ is open with respect to the topology $\mathcal{G}$ if and only if $\left\{X_{j}: j \in A\right\}$ is open in $\mathcal{X}$ with respect to the weak topology. It is known that $\mathcal{X}$ with the weak topology is a separable metric space (see, for example, [12, p. 122]), therefore, $(J, \mathcal{g})$ is also a separable metric space, and these two spaces are isometric. Therefore, $\left(j_{n}\right)$ converges to $j$ (in the topology $g$ ) if and only if $\left(X_{j_{n}}\right)$ converges in distribution to $X_{j}$.

Lemma 2, below, tells us that the total order on $X$ is continuous in the sense that

$$
\left\{X_{j} \in \mathcal{X}: X_{j}<_{\text {st }} X_{i}\right\} \quad \text { and } \quad\left\{X_{j} \in \mathcal{X}: X_{i}<_{\text {st }} X_{j}\right\}
$$

are open subsets of $\mathcal{X}$ for every $X_{i} \in \mathcal{X}$. 
Lemma 2. The total order on $\mathcal{X}$ defined above is continuous with respect to the weak topology on $\mathcal{X}$.

Proof. We first show that the subset $A=\left\{X_{j} \in \mathcal{X}: X_{j} \leq_{\text {st }} X_{i}\right\}$ is closed in the weak topology for every fixed $X_{i} \in \mathcal{X}$. Let $\left(X_{j_{n}}\right)$ be a sequence in $A$ that converges in distribution to $X_{0} \in X$. Clearly, $F_{j_{n}}(u) \geq F_{i}(u)$ for all $u \in \mathbb{R}$ and for all $n \in \mathbb{N}$, and $F_{j_{n}}(u) \rightarrow F_{0}(u)$ when $u$ is a continuity point of $F_{0}$. Therefore, $F_{0}(u) \geq F_{i}(u)$ for all $u$, meaning that $X_{0} \in A$ as well. This proves that $A$ is closed and, hence, the complement $\left\{X_{j} \in \mathcal{X}: X_{i}<_{\text {st }} X_{j}\right\}$ is open. By the same argument, a subset of the form $\left\{X_{j} \in \mathcal{X}: X_{j}<_{\text {st }} X_{i}\right\}$ is also open.

An equivalent way to state this result is that the order topology on $\mathcal{X}$ is weaker than the weak topology. As a direct consequence of Lemma 2, the set $\left\{\left(X_{j}, X_{i}\right): X_{j}<_{\text {st }} X_{i}\right\}$ is open in $X^{2}$ (equipped with the product topology), while $\left\{\left(X_{j}, X_{i}\right): X_{j} \leq_{\text {st }} X_{i}\right\}$ is closed (see, for example, [6]).

Assumption (c), which states that the mixing variables are independent, will make the problem analytically more tractable. Assumption (d) guarantees the measurability of each $X_{M_{i}}$. Assumption (e) is self explanatory. Because of this assumption, the investor will first minimize the expected utility over all possible dependence structures in $\left\{X_{j}: j \in J\right\}$ before constructing the optimal portfolio. Therefore, the original problem becomes the following problem.

Problem 1. Solve

$$
\max _{\boldsymbol{a} \in \S(W)} \min _{X} \mathrm{E}\left[U\left(\sum_{i=1}^{n} a_{i} X_{M_{i}}\right)\right]
$$

\section{Worst dependence structure}

In this section we will solve the 'min' part of Problem 1, that is, we will identify the worst dependence structure that minimizes the expected utility.

Proposition 1. Let $\left\{X_{j}^{\mathrm{c}}: j \in J\right\}$ be a comonotonic copy of $\left\{X_{j}: j \in J\right\}$ that is independent of $M_{1}, \ldots, M_{n}$, and is jointly measurable. Then

$$
\mathrm{E}\left[U\left(\sum_{i=1}^{n} a_{i} X_{M_{i}}^{\mathrm{c}}\right)\right] \leq \mathrm{E}\left[U\left(\sum_{i=1}^{n} a_{i} X_{M_{i}}\right)\right]
$$

for any $\left(a_{1}, \ldots, a_{n}\right) \in \&(W)$.

Proof. Fix any $j_{1}, \ldots, j_{n} \in J$. The collection $\left\{X_{j_{1}}^{\mathrm{c}}, \ldots, X_{j_{n}}^{\mathrm{c}}\right\}$ is comonotonic by hypothesis. Since each $a_{i}$ is nonnegative, the collection $\left\{-a_{1} X_{j_{1}}^{\mathrm{c}}, \ldots,-a_{n} X_{j_{n}}^{\mathrm{c}}\right\}$ is also comonotonic. Therefore,

$$
-\sum_{i=1}^{n} a_{i} X_{j_{i}} \leq_{\mathrm{cx}}-\sum_{i=1}^{n} a_{i} X_{j_{i}}^{\mathrm{c}}
$$

and, hence,

$$
\mathrm{E}\left[U\left(\sum_{i=1}^{n} a_{i} X_{j_{i}}\right)\right] \geq \mathrm{E}\left[U\left(\sum_{i=1}^{n} a_{i} X_{j_{i}}^{\mathrm{c}}\right)\right],
$$

because $x \mapsto-U(-x)$ is convex. The result follows by taking the conditional expectation of $M_{1}, \ldots, M_{n}$ to both sides of this inequality. 
Owing to this result, from now on we will assume that $\left\{X_{j}: j \in J\right\}$ is comonotonic, in addition to the standing assumptions made in the previous section. The problem of the existence of such a collection is dealt with in the next section. One important consequence is that if $j_{1} \preceq j_{2}$ then $X_{j_{1}} \leq_{\text {st }} X_{j_{2}}$ and, hence, $X_{j_{1}} \leq X_{j_{2}}$ on $\Omega$ by comonotonicity. In particular, this means that every realization of $\left\{X_{j}: j \in J\right\}$ is an increasing real-valued function on $J$.

\section{Existence of a comonotonic and jointly measurable collection}

In view of the characterization of the worst dependence structure described in Section 3, the next important problem we should study is the problem of existence.

Problem. (Problem of existence.) Given a set of distinct marginal distributions $\left\{F_{j}: j \in J\right\}$, is there a stochastic process $\left\{X_{j}: j \in J\right\}$ that is comonotonic, jointly measurable, and has the prescribed marginal distributions?

Based on our standing assumptions, the collection $\left\{F_{j}: j \in J\right\}$ should satisfy the following assumption.

Assumption 1. When a sequence $\left(j_{n}\right)$ in $J$ converges to $j$ in the topology $\mathcal{G},\left(F_{j_{n}}\right)$ converges to $F_{j}$ at all continuity points of $F_{j}$.

To solve the problem of existence, we need the following general result concerning the existence of a measurable modification of a stochastic process, which is taken from [8].

Lemma 3. Let $\left\{X_{t}: t \in T\right\}$ be a stochastic process defined on $(\Omega, \mathcal{F}, P)$ with values in $K$, in which the index set $T$ is a separable metric space and $K$ is a compact metric space. Set $Q(s, t, A)=\mathrm{P}\left(\left(X_{s}, X_{t}\right) \in A\right)$ for $(s, t) \in T^{2}$ and $A \in \mathscr{B}\left(K^{2}\right)$. Suppose that $g$ is a subset of $\mathscr{B}(K)$ which is closed under finite intersection such that $\sigma\{g\}=\mathscr{B}(K)$. Then $\left\{X_{t}: t \in T\right\}$ has a measurable modification if and only if the following two conditions hold.

1. The map $t \mapsto Q(s, t, G \times H)$ is a measurable map from $T$ into $[0,1]$ for all $s \in T$ and $G, H \in g$.

2. There exists a countable set $T_{0} \subseteq T$ such that, for all $t \in T$, we can find a sequence $\left(t_{n}\right)$ in $T_{0}$ with $Q\left(t_{n}, t, \cdot\right)$ converging weakly to $Q(t, t, \cdot)$.

Proposition 2. If $\left\{F_{j}: j \in J\right\}$ is a given set of distinct distribution functions that satisfies Assumption 1 then there exists a stochastic process $\left\{X_{j}: j \in J\right\}$ that is comonotonic, jointly measurable, and $X_{j} \sim F_{j}$ for all $j$.

Proof. We first construct a process that is comonotonic and has the prescribed marginal distributions. Let $U$ be an arbitrary uniform $(0,1)$ random variable. For each $j \in J$, we define $\tilde{X}_{j}=F_{j}^{-1}(U)$. Clearly, $\tilde{X}_{j} \sim F_{j}$ for all $j$, and $\left\{\tilde{X}_{j}: j \in J\right\}$ is comonotonic.

Next we show that the process $\left\{\tilde{X}_{j}: j \in J\right\}$ has a measurable modification using Lemma 3 . This measurable modification is exactly the process we want. To this end, we recall that our index set $J$ with the topology $g$ is a separable metric space, and we use the one-point compactification of $\mathbb{R}$ as the state space instead of using $\mathbb{R}$ directly. We denote this onepoint compactification as $\overline{\mathbb{R}}$. Let $g=\{(-\infty, s]: s \in \mathbb{R}\}$. Obviously, $g$ is closed under finite intersection, and the $\sigma$-field generated by it is $\mathcal{B}(\overline{\mathbb{R}})$. In order to prove that the process $\left\{\tilde{X}_{j}: j \in J\right\}$ has a measurable modification, we need to verify the two conditions stated in Lemma 3. 
For condition 1 of Lemma 3, we need to verify the measurability of the map $j \mapsto f(j)=$ $\mathrm{P}\left(\tilde{X}_{i} \leq s, \tilde{X}_{j} \leq t\right)$ for any fixed $i \in J$ and for any fixed real numbers $s$ and $t$. Since $\left(\tilde{X}_{i}, \tilde{X}_{j}\right)$ is comonotonic, it follows from (1) that

$$
f(j)=\mathrm{P}\left(\tilde{X}_{i} \leq s, \tilde{X}_{j} \leq t\right)=\min \left(\mathrm{P}\left(\tilde{X}_{i} \leq s\right), \mathrm{P}\left(\tilde{X}_{j} \leq t\right)\right) .
$$

By Assumption 1 and the well-known Portmanteau theorem on weak convergence (see, for example, [1, p. 11]), the map $j \mapsto \mathrm{P}\left(\tilde{X}_{j} \in(-\infty, t]\right)$ is lower semicontinuous because $(-\infty, t]$ is a closed set. Hence, this map is measurable as well. This in turn implies the measurability of the map $f$.

For condition 2 of Lemma 3, we first select a countable dense subset $J_{0}$ in $J$ by the separability. For any $j \in J$, there exists a sequence $\left\{j_{n}\right\} \subseteq J_{0}$ that converges to $j$. We need to show that $\left(\tilde{X}_{j_{n}}, \tilde{X}_{j}\right)$ converges in distribution to $\left(\tilde{X}_{j}, \tilde{X}_{j}\right)$. However, this is just an immediate consequence of our construction: when $j_{n}$ converges to $j, \tilde{X}_{j_{n}}$ converges almost surely to $\tilde{X}_{j}$ by Assumption 1 and, hence, $\left(\tilde{X}_{j_{n}}, \tilde{X}_{j}\right)$ also converges almost surely to $\left(\tilde{X}_{j}, \tilde{X}_{j}\right)$. Convergence in distribution then follows.

We remark that the process $\left\{\tilde{X}_{j}: j \in J\right\}$ constructed in the first step of the above proof is indeed the Skorohod representation of a sequence of weakly convergent random variables. This enables us to transfer weak convergence to almost sure convergence in the second step, which is the crux of the proof. Proposition 2 also explains the reason why we adopt the topology $g$ on $J$ instead of the order topology, which is weaker than $g$ by Lemma 2 . If the weaker order topology was used then the second step of the above proof is no longer valid. Of course, the cost of this convenience is that each $M_{i}: \Omega \rightarrow J$ has to be measurable with respect to a larger $\sigma$-field on $J$.

Finally, we note that by choosing the uniform $(0,1)$ random variable $U$ to be independent of $M_{1}, \ldots, M_{n}$, then the collection constructed in this proof will also be independent of $M_{1}, \ldots, M_{n}$.

\section{Main result}

In this section we are going to obtain conditions that allow us to rank the optimal allocations by comparing the mixing variables representing the securities. Intuitively, if $M_{i}$ is relatively large (recall that $J$ is totally ordered) then the return of the $i$ th security will also be relatively large because the usual stochastic order among $\left\{X_{j}: j \in J\right\}$ is consistent with the order in $J$. To make this precise and to prepare for our main result, we need the following definition.

Definition 1. Let $W$ and $Y$ be two measurable maps in $J$. Then $W$ is said to be smaller than $Y$ in the likelihood ratio order if

$$
\mathrm{P}(W \in S) \mathrm{P}(Y \in T) \geq \mathrm{P}(W \in T) \mathrm{P}(Y \in S)
$$

for all measurable subsets $S$ and $T$ in $J$ such that $s \in S$ and $t \in T$ imply that $s \preceq t$ (i.e. $S \preceq T$ ). In this case we write $W \leq \operatorname{lr} Y$.

Note that this definition is equivalent to the classical definition when $J$ is the real line equipped with the usual order on $\mathbb{R}$. For more information on the likelihood ratio order, we refer the reader to [11].

Theorem 1. Suppose that $\boldsymbol{a}^{*}=\left(a_{1}^{*}, \ldots, a_{n}^{*}\right)$ is the solution to Problem 1. Then

$$
M_{1} \leq \operatorname{lr} M_{2} \Longrightarrow a_{1}^{*} \leq a_{2}^{*} \text {. }
$$


The proof of this result requires several lemmas. We first introduce some notation. Let $x=\left\{x_{j}: j \in J\right\}$ be an increasing real-valued function on $J$ (i.e. $j_{1} \preceq j_{2} \Rightarrow x_{j_{1}} \leq x_{j_{2}}$ ), and let $a_{1} \leq a_{2}$ be two nonnegative numbers. We define the following subsets of $J^{2}$ :

$$
\begin{array}{rlrl}
L\left(x, a_{1}, a_{2} ; u\right) & =\left\{\left(j_{1}, j_{2}\right) \in J^{2}: a_{1} x_{j_{1}}+a_{2} x_{j_{2}}<u\right\}, & & u \in \mathbb{R}, \\
R\left(x, a_{1}, a_{2} ; u\right) & =\left\{\left(j_{1}, j_{2}\right) \in J^{2}: a_{2} x_{j_{1}}+a_{1} x_{j_{2}}<u\right\}, & & u \in \mathbb{R}, \\
A & =\left\{\left(j_{1}, j_{2}\right) \in J^{2}: j_{1} \prec j_{2}\right\}, & \\
B & =\left\{\left(j_{1}, j_{2}\right) \in J^{2}: j_{2} \prec j_{1}\right\}, & \\
C & =\left\{\left(j_{1}, j_{2}\right) \in J^{2}: j_{1}=j_{2}\right\} . &
\end{array}
$$

Moreover, for any subset $D$ of $J^{2}$, its reflection $\left\{\left(j_{1}, j_{2}\right) \in J^{2}:\left(j_{2}, j_{1}\right) \in D\right\}$ is denoted as $\bar{D}$. Note that $A, B$, and $C$ form a partition of $J^{2}$ because $J$ is totally ordered. It is also noted that $A$ and $B$ are open in $J^{2}$ (equipped with the product topology), while $C$ is closed.

Lemma 4. Let $x=\left\{x_{j}: j \in J\right\}$ be an increasing real-valued function on $J$, and let $a_{1} \leq a_{2}$ be two nonnegative numbers. Then

1. $R\left(x, a_{1}, a_{2} ; u\right) \cap B \subseteq L\left(x, a_{1}, a_{2} ; u\right) \cap B ;$

2. $L\left(x, a_{1}, a_{2} ; u\right) \cap A \subseteq R\left(x, a_{1}, a_{2} ; u\right) \cap A$;

3. $L\left(x, a_{1}, a_{2} ; u\right) \cap C=R\left(x, a_{1}, a_{2} ; u\right) \cap C$;

4. $\overline{\left(L\left(x, a_{1}, a_{2} ; u\right) \backslash R\left(x, a_{1}, a_{2} ; u\right)\right) \cap B}=\left(R\left(x, a_{1}, a_{2} ; u\right) \backslash L\left(x, a_{1}, a_{2} ; u\right)\right) \cap A$.

Proof. Suppose that $\left(j_{1}, j_{2}\right) \in R\left(x, a_{1}, a_{2} ; u\right) \cap B$. Then $j_{2} \prec j_{1}$ and $a_{2} x_{j_{1}}+a_{1} x_{j_{2}}<u$. Since $x$ is increasing, $j_{2} \prec j_{1}$ implies that $x_{j_{2}} \leq x_{j_{1}}$. Hence,

$$
a_{1} x_{j_{1}}+a_{2} x_{j_{2}} \leq a_{2} x_{j_{1}}+a_{1} x_{j_{2}}<u,
$$

by rearranging the inequality. Therefore, $\left(j_{1}, j_{2}\right) \in L\left(x, a_{1}, a_{2} ; u\right)$ as well. This proves the first relationship. The other relationships can be proved similarly and are omitted.

Lemma 5, below, deals with the comparison of two measures by comparing their sizes on a generating semiring.

Lemma 5. Suppose that $\mu_{1}$ and $\mu_{2}$ are two $\sigma$-finite measures on a measurable space $(Y, \mathcal{y})$, and $\mathcal{R}$ is a semiring that generates the $\sigma$-field $\mathcal{y}$. If $\mu_{1} \geq \mu_{2}$ on $\mathcal{R}$ the $\mu_{1} \geq \mu_{2}$ on $\mathcal{y}$.

Proof. Let $\mu_{1}^{*}$ and $\mu_{2}^{*}$ be the Carathéodory extensions of $\mu_{1}$ and $\mu_{2}$, respectively, that is,

$$
\mu_{i}^{*}(D)=\inf \left\{\sum_{k=1}^{\infty} \mu_{i}\left(D_{k}\right): D_{k} \in \mathcal{R} \text { for all } k \text { and } D \subseteq \bigcup_{k=1}^{\infty} D_{k}\right\}, \quad i=1,2, D \in 2^{Y}
$$

Obviously, $\mu_{1} \geq \mu_{2}$ on $\mathcal{R}$ implies that $\mu_{1}^{*} \geq \mu_{2}^{*}$ on the power set $2^{Y}$. Since $\mu_{1}$ and $\mu_{2}$ are the restrictions of $\mu_{1}^{*}$ and $\mu_{2}^{*}$ to $\mathcal{Y}$, respectively, the result follows.

A subset $D$ of $J^{2}$ is called a measurable rectangle if it can be expressed as $D=S \times T$ for some measurable subsets $S$ and $T$ of $J$. The collection of all measurable rectangles that are contained in $A$ will be denoted as $\mathcal{R}_{A}$. Lemma 6, below, demonstrates some properties of $\mathcal{R}_{A}$. 
Lemma 6. The collection $\mathcal{R}_{A}$ is a semiring in $A$ and it generates the $\sigma$-field $(\mathscr{B}(J) \otimes \mathscr{B}(J)) \cap$ $A=\mathscr{B}\left(J^{2}\right) \cap A$.

Proof. The semiring property is obvious. The last equality follows from the second countability of the topology $\mathcal{g}$. It is also clear that

$$
\sigma\left\{\mathcal{R}_{A}\right\} \subseteq(\mathcal{B}(J) \otimes \mathscr{B}(J)) \cap A
$$

To obtain the reverse inclusion, it is enough to note that $J^{2}$ with the product topology is second countable, and, hence, any open subset $G$ of $J^{2}$ contained in $A$ can be expressed as a union of countably many measurable rectangles $S_{n} \times T_{n} \in \mathcal{R}_{A}$, where each $S_{n}$ and $T_{n}$ is open in $J$.

The following result provides a characterization of the likelihood ratio order. For each $i=1,2, \ldots, n$, we denote the distribution of $M_{i}$ on $(J, \mathscr{B}(J))$ by $\mathbb{M}_{i}$.

Lemma 7. We have $M_{1} \leq$ lr $M_{2}$ if and only if $\mathbb{M}_{1} \otimes \mathbb{M}_{2}(D) \geq \mathbb{M}_{2} \otimes \mathbb{M}_{1}(D)$ for any $D \in$ $\mathcal{B}\left(J^{2}\right) \cap A$.

Proof. Let $D=S \times T$ be an arbitrary measurable rectangle contained in $A$. Then $S \preceq T$ and, hence,

$$
\begin{aligned}
\mathbb{M}_{1} \otimes \mathbb{M}_{2}(D) \geq \mathbb{M}_{2} \otimes \mathbb{M}_{1}(D) & \Longleftrightarrow \mathrm{P}\left(\left(M_{1}, M_{2}\right) \in D\right) \geq \mathrm{P}\left(\left(M_{2}, M_{1}\right) \in D\right), \\
& \Longleftrightarrow \mathrm{P}\left(M_{1} \in S\right) \mathrm{P}\left(M_{2} \in T\right) \geq \mathrm{P}\left(M_{2} \in S\right) \mathrm{P}\left(M_{1} \in T\right), \\
& \Longleftrightarrow M_{1} \leq \text { lr } M_{2} \quad \text { as } S \preceq T \text { are arbitrary. }
\end{aligned}
$$

This proves the if part and also the only if part when $D \in \mathcal{R}_{A}$. In other words, $M_{1} \leq_{\operatorname{lr}} M_{2}$ implies that $\mathbb{M}_{1} \otimes \mathbb{M}_{2} \geq \mathbb{M}_{2} \otimes \mathbb{M}_{1}$ on $\mathcal{R}_{A}$. Combining Lemmas 5 and 6 , we conclude that $\mathbb{M}_{1} \otimes \mathbb{M}_{2} \geq \mathbb{M}_{2} \otimes \mathbb{M}_{1}$ on $\mathcal{B}\left(J^{2}\right) \cap A$.

Using the above characterization, we can prove the following result, which is basically the probabilistic version of the well-known Hardy-Littlewood-Pólya rearrangement inequality.

Lemma 8. Let $x=\left\{x_{j}: j \in J\right\}$ be an increasing and measurable real-valued function on $J$, and let $a_{1} \leq a_{2}$ be two nonnegative numbers. Then $M_{1} \leq \mathrm{rr} M_{2}$ implies that

$$
a_{1} x_{M_{1}}+a_{2} x_{M_{2}} \geq_{\mathrm{st}} a_{2} x_{M_{1}}+a_{1} x_{M_{2}} .
$$

Proof. Let $u$ be any fixed real number. To simplify the notation, we will denote the lefthand side and the right-hand side by $L$ and $R$, respectively; moreover, $L\left(x, a_{1}, a_{2} ; u\right)$ and $R\left(x, a_{1}, a_{2} ; u\right)$ will be simplified as $L(u)$ and $R(u)$, respectively. We note that both $L(u)$ and $R(u)$ are measurable subsets of $J^{2}$ because $x=\left\{x_{j}: j \in J\right\}$ is measurable. Hence,

$$
\begin{aligned}
\mathrm{P}(R<u) & =\mathrm{P}\left(\left(M_{1}, M_{2}\right) \in R(u)\right) \\
& =\mathbb{M}_{1} \otimes \mathbb{M}_{2}(R(u)) \\
& =\mathbb{M}_{1} \otimes \mathbb{M}_{2}(R(u) \cap A)+\mathbb{M}_{1} \otimes \mathbb{M}_{2}(R(u) \cap B)+\mathbb{M}_{1} \otimes \mathbb{M}_{2}(R(u) \cap C)
\end{aligned}
$$

Similarly, we have

$$
\mathrm{P}(L<u)=\mathbb{M}_{1} \otimes \mathbb{M}_{2}(L(u) \cap A)+\mathbb{M}_{1} \otimes \mathbb{M}_{2}(L(u) \cap B)+\mathbb{M}_{1} \otimes \mathbb{M}_{2}(L(u) \cap C) .
$$


Then, by Lemma 4,

$$
\begin{aligned}
\mathrm{P}(R<u)-\mathrm{P}(L<u)= & \mathbb{M}_{1} \otimes \mathbb{M}_{2}(R(u) \cap A)+\mathbb{M}_{1} \otimes \mathbb{M}_{2}(R(u) \cap B) \\
& -\mathbb{M}_{1} \otimes \mathbb{M}_{2}(L(u) \cap A)-\mathbb{M}_{1} \otimes \mathbb{M}_{2}(L(u) \cap B) \\
= & \mathbb{M}_{1} \otimes \mathbb{M}_{2}((R(u) \backslash L(u)) \cap A)-\mathbb{M}_{1} \otimes \mathbb{M}_{2}((L(u) \backslash R(u)) \cap B) \\
= & \mathbb{M}_{1} \otimes \mathbb{M}_{2}((R(u) \backslash L(u)) \cap A)-\mathbb{M}_{2} \otimes \mathbb{M}_{2}(\overline{(L(u) \backslash R(u)) \cap B}) \\
= & \mathbb{M}_{1} \otimes \mathbb{M}_{2}((R(u) \backslash L(u)) \cap A)-\mathbb{M}_{2} \otimes \mathbb{M}_{2}((R(u) \backslash L(u)) \cap A) \\
\geq & 0,
\end{aligned}
$$

where the last inequality follows from Lemma 7. The proof is complete.

We remark that the probabilistic rearrangement inequality presented in [9, Theorem 4.9] is a special case of Lemma 8 when $J$ is the real line equipped with the usual order on $\mathbb{R}$ and $x_{j}=j$.

Proof of Theorem 1. Suppose that $M_{1} \leq_{\operatorname{lr}} M_{2}$. Fix any $\boldsymbol{a}=\left(a_{1}, \ldots, a_{n}\right) \in \delta(W)$ with $a_{1} \leq a_{2}$. Define $\boldsymbol{a}^{\prime}=\left(a_{1}^{\prime}, a_{2}^{\prime}, a_{3}^{\prime}, \ldots, \overline{a_{n}^{\prime}}\right)=\left(a_{2}, a_{1}, a_{3}, \ldots, a_{n}\right) \in \delta(W)$. We also fix $x=\left\{x_{j}: j \in J\right\}$ as a realization (sample path) of $\left\{X_{j}: j \in J\right\}$. Then $x=\left\{x_{j}: j \in J\right\}$ is an increasing and measurable real-valued function on $J$. By Lemma 8 we obtain

$$
a_{1} x_{M_{1}}+a_{2} x_{M_{2}} \geq_{\mathrm{st}} a_{2} x_{M_{1}}+a_{1} x_{M_{2}} .
$$

As the increasing concave order is weaker than the usual stochastic order and as it is closed under convolution with independent variables, we obtain

$$
\sum_{i=1}^{n} a_{i} x_{M_{i}} \geq_{\mathrm{icv}} \sum_{i=1}^{n} a_{i}^{\prime} x_{M_{i}}
$$

which implies that

$$
\mathrm{E}\left[U\left(\sum_{i=1}^{n} a_{i} x_{M_{i}}\right)\right] \geq \mathrm{E}\left[U\left(\sum_{i=1}^{n} a_{i}^{\prime} x_{M_{i}}\right)\right] .
$$

By taking the expectation conditional of $\left\{X_{j}: j \in J\right\}$ to both sides of this inequality, we conclude that

$$
\mathrm{E}\left[U\left(\sum_{i=1}^{n} a_{i} X_{M_{i}}\right)\right] \geq \mathrm{E}\left[U\left(\sum_{i=1}^{n} a_{i}^{\prime} X_{M_{i}}\right)\right] .
$$

The result follows from this inequality.

If we examine the whole argument carefully, the following is clear: in order to compare the size of the optimal amount invested in two different securities, say the $i$ th and the $j$ th, we do not need a total order on the whole index set $J$ or, equivalently, we do not need a total order on $\left\{X_{j}: j \in J\right\}$. It is sufficient to have a total order on $\operatorname{Range}\left(M_{i}\right) \cup \operatorname{Range}\left(M_{j}\right)$. It should also be remarked that the concavity of the utility function $U$ is only used in establishing the worst dependence structure.

We close this section by presenting a simple illustrating example.

Example. Suppose that $J=\{1,2\}$ such that $1 \prec 2$, where $\{1\}$ corresponds to a 'bad' environment and $\{2\}$ corresponds to a 'good' environment. Assume that $X_{1} \sim \log N(\mu, \sigma)$ and $X_{2} \sim \log N(\nu, \sigma)$, where $\mu \leq \nu$, and assume that they have unknown dependence structure. 
It can be checked that $X_{1} \leq_{\text {st }} X_{2}$. Consider the independent securities $M_{1}=(0.7,0.3)$ and $M_{2}=(0.4,0.6)$. Obviously, the second security is better because it has a higher probability (0.6) of being in a good environment to enjoy a better investment return. In fact, direct calculation shows that $M_{1} \leq \mathrm{lr} M_{2}$. Hence, by Theorem 1 , we should invest more in the second security than in the first.

\section{Special cases}

The model studied in this paper is indeed quite general in the sense that it includes several models studied in the literature before as special cases. In the next two examples we will explain this relationship, which serve as applications of our current model.

Example 1. Suppose that $J$ is a finite set, say $J=\{1,2, \ldots, n\}$. Then there are $n$ fundamental risks $X_{1}, \ldots, X_{n}$. Furthermore, suppose that all the mixing variables are degenerate such that $M_{i} \equiv i$ for all $i$. Then it is trivial to show that $M_{1}, \ldots, M_{n}$ are independent of each other and independent of $X_{1}, \ldots, X_{n}$. Therefore, the setting here satisfies all the assumptions required in our mixture model. The optimization problem becomes

$$
\max _{\boldsymbol{a} \in S(W)} \min _{X} \mathrm{E}\left[U\left(\sum_{i=1}^{n} a_{i} X_{i}\right)\right] .
$$

The interpretation of this model is that the return of securities is regarded as the fundamental risk in the market. This problem was studied in [2]. By applying our analysis we obtain the same conclusion as in that paper: $X_{i} \leq_{\mathrm{st}} X_{j} \Rightarrow a_{i}^{*} \leq a_{j}^{*}$.

Example 2. Suppose that $J=\mathbb{R}$ is endowed with the usual order of real numbers. One way to introduce the system of fundamental risks $\left\{X_{j}: j \in \mathbb{R}\right\}$ that is totally ordered and is consistent with the usual order on $\mathbb{R}$ is to set $X_{j} \equiv j$ for all $j$. Since every $X_{j}$ is degenerate, the worst dependence structure among them is meaningless. Moreover, we note that the topology $g$ on $J$ (induced from the weak topology on $X$ ) is the same as the usual Euclidean topology because $\left(X_{j_{n}}\right)$ converges in distribution to $X_{j}$ if and only if $\left(j_{n}\right)$ converges to $j$ in the Euclidean topology. Hence, the optimization becomes

$$
\max _{\boldsymbol{a} \in \mathcal{S}(W)} \mathrm{E}\left[U\left(\sum_{i=1}^{n} a_{i} M_{i}\right)\right] .
$$

Since there is no need to establish the worst dependence structure, the concave assumption on $U$ can be relaxed. This problem was studied in [9]. By applying the bivariate characterization of the likelihood ratio order, they first obtained a stochastic version of the rearrangement inequality, and then deduced that $M_{i} \leq \operatorname{lr} M_{j} \Rightarrow a_{i}^{*} \leq a_{j}^{*}$, which is consistent with Theorem 1 .

\section{Acknowledgements}

We would like to thank an anonymous referee for helpful suggestions and comments. Ka Chun Cheung acknowledges financial support from the Natural Sciences and Engineering Research Council of Canada. Hailiang Yang acknowledges financial support from the Research Grants Council of HKSAR (project number HKU 7426/06H). 


\section{References}

[1] Billingsley, P. (1968). Convergence of Probability Measures. John Wiley, New York.

[2] Cheung, K. C. (2006). Optimal portfolio problem with unknown dependency structure. Insurance Math. Econom. 38, 167-175.

[3] Cheung, K. C. (2007). Optimal allocation of policy limits and deductibles. Insurance Math. Econom. 41, 382-391.

[4] Cheung, K. C. And Yang, H. (2004). Ordering optimal proportions in the asset allocation problem with dependent default risks. Insurance Math. Econom. 35, 595-609.

[5] Dhaene, J. et al. (2002). The concept of comonotonicity in actuarial science and finance: theory. Insurance Math. Econom. 31, 3-33.

[6] Föllmer, H. And Schied, A. (2004). Stochastic Finance. An Introduction in Discrete Time (De Gruyter Studies Math. 27). Walter de Gruyter, Berlin.

[7] Hennessy, D. A. And Lapan, H. E. (2002). The use of Archimedean copulas to model portfolio allocations. Math. Finance 12, 143-154.

[8] Hoffmann-Jørgensen, J. (1973). Existence of measurable modifications of stochastic processes. Z. Wahrscheinlichkeitsth. 25, 205-207.

[9] KiJima, M. AND OHNishi, M. (1996). Portfolio selection problems via the bivariate characterization of stochastic dominance relations. Math. Finance 6, 237-277.

[10] Lapan, H. E. ANd Hennessy, D. A. (2002). Symmetry and order in the portfolio allocation problem. Econom. Theory 19, 747-772.

[11] Shaked, M. and Shanthikumar, J. G. (1994). Stochastic Orders and Their Applications. Academic Press, Boston, MA.

[12] Stroock, D. W. (1993). Probability Theory, An Analytic View. Cambridge University Press. 